\title{
Human \& swine studies of concurrent 12-lead ECG \& MRI
}

\author{
Zion Tse ${ }^{1 *}$, Charles Dumoulin ${ }^{2}$, Ronald Watkins ${ }^{3}$, Kim Butts Pauly ${ }^{3}$, Israel Byrd ${ }^{4}$, Jeffrey Schweitzer ${ }^{4}$, \\ Raymond Y Kwong ${ }^{5}$, Gregory F Michaud ${ }^{5}$, William Stevenson ${ }^{5}$, Ferenc Jolesz ${ }^{6}$, Ehud J Schmidt ${ }^{6}$ \\ From 16th Annual SCMR Scientific Sessions \\ San Francisco, CA, USA. 31 January - 3 February 2013
}

\section{Background}

12-lead Electrocardiogram (ECG) is a clinical standard for patient physiological monitoring. An MRI-conditional 12-lead ECG should permit detection of acute myocardial ischemia during MR imaging or MRI-guided therapy, which may improve the handling of patients with ischemic histories. MRI visualization of ischemic episodes can also enhance the understanding of ischemic progression. Previously an MR-conditional 12-lead ECG system was presented. The system was equipped with GradientRamp\&RF (GR\&RF) noise removal hardware \& Magnetohydrodynamic (VMHD) voltage-removal software that improved ST segment visualization [1]. The study objectives were to (1) validate simultaneous 12-lead ECG monitoring \& cardiac MR imaging in human subjects; (2) detect $\mathrm{S}$-wave to $\mathrm{T}$-wave (ST) ECG elevation \& perform MR imaging of a Left Anterior Descending (LAD) balloon occlusion from the onset of ischemia to death in a swine model.

\section{Methods}

1) Human Studies (Fig1) - 12-lead ECG \& MRI in 14 subjects (including 2 premature ventricular contraction \& 2 atrial fibrillation (AF) patients): Cardiac imaging was performed in a GE 1.5T MRI, with scans triggered by the 12-lead ECG \& with simultaneous ECGs recording (1a-d). The derived real ECGs were compared to ECGs measured outside MRI for validation (1e-f).

2) Swine Study (Fig2) - Acute Ischemia Progressing to Death: A 2-mm balloon catheter filled with Gadoliniumdoped water was inserted into the swine's distal LAD using X-ray guidance. The swine was moved to the MRI where continuous 12-lead ECG monitoring (2a) and cine

'Engineering, The University of Georgia, Athens, GA, USA

Full list of author information is available at the end of the article imaging ( $2 \mathrm{~b}-\mathrm{d}$ ) were performed. At $\mathrm{t}=0$ seconds, the balloon was inflated to 20 atmospheres. MRI \& simultaneous ECG monitoring were maintained until death $~ 20$ minutes later. The balloon's position was confirmed using post-mortem 3D T1 imaging, \& T2 imaging was used to detect edema.

\section{Results}

1) AF patient's ECG processing \& MRI (Fig1): (a) Raw ECG V6 was dominated by GR\&RF noise during a GRE scan. (b) GR\&RF noise was removed using the hardware circuit, leaving real ECG superimposed with VMHD. (c) Real ECG was extracted. (d) Beat-to-beat stroke volume (BTB-SV) was estimated from VMHD, where varying ventricular filling due to changing heart rates is responsible for irregular BTB-SV results. ST segment was well preserved for ischemia monitoring (c, e-f). The system outputted $100 \%$ accurate scan triggers at $<30 \mathrm{~ms}$ latency, allowing cine MRI in subjects where 4-lead ECG gating failed due to stronger VMHD peaks (g).

2) Acute Ischemia in Swine (Fig2): ST elevation was detected 1.5 seconds after onset (a1), progressing to acute ischemia (a2-3), bradycardia and death. Ventricular dysfunction and unusual flow vortexes were visualized with serial cine MRI (c1-4). Epicardial edema was observed adjacent to the balloon (d).

\section{Conclusions}

MRI-conditional 12-lead ECG provides high-fidelity ECGs for robust cardiac-MRI. Acute ischemia detection is possible, with simultaneous MRI visualization of dysfunction progression.

\section{Funding}

NIH U41-RR019703, R43 HL110427-01, AHA 10SD G261039. 


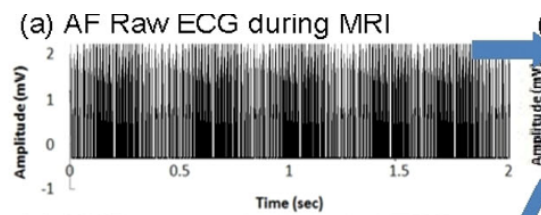

(c) $\mathrm{MHD}$ removed-extracted $E \mathrm{CG}_{\text {real }}$

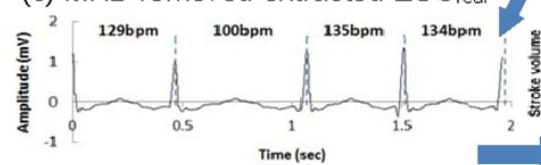

(e) ST Elevation: Mean, Maximum \& (f) ST Elevation: Standard Deviation Minimum

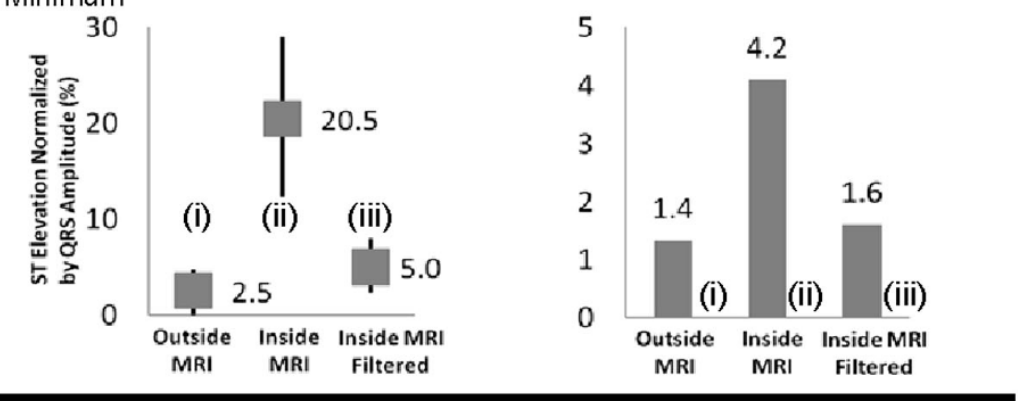

(g) 12-lead ECG-gated cardiac cine
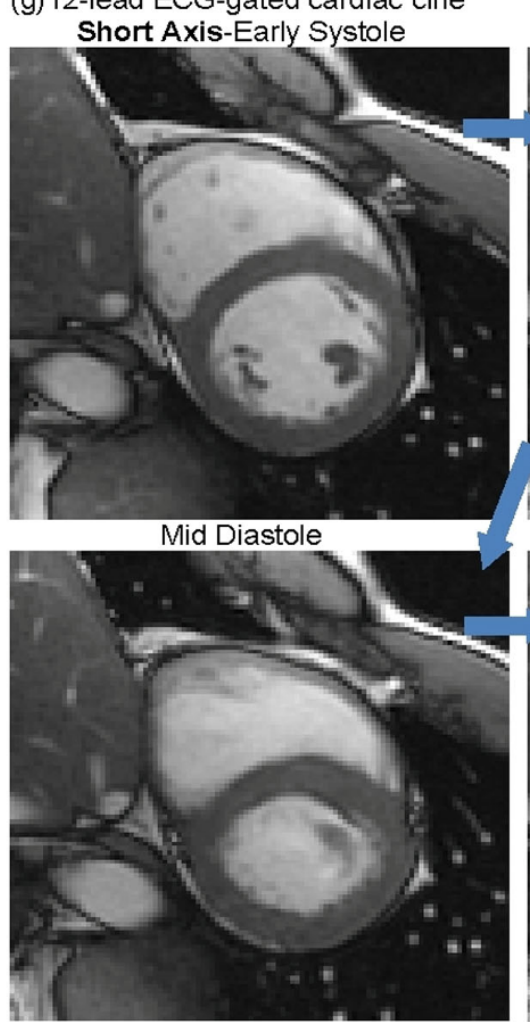

(b) Switching enabled( $\left(\mathrm{CG}_{\text {real }}+\mathrm{MHD}\right)$

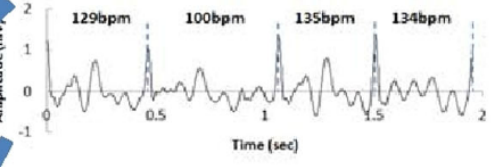

(d) Beat-To-Beat stroke volume
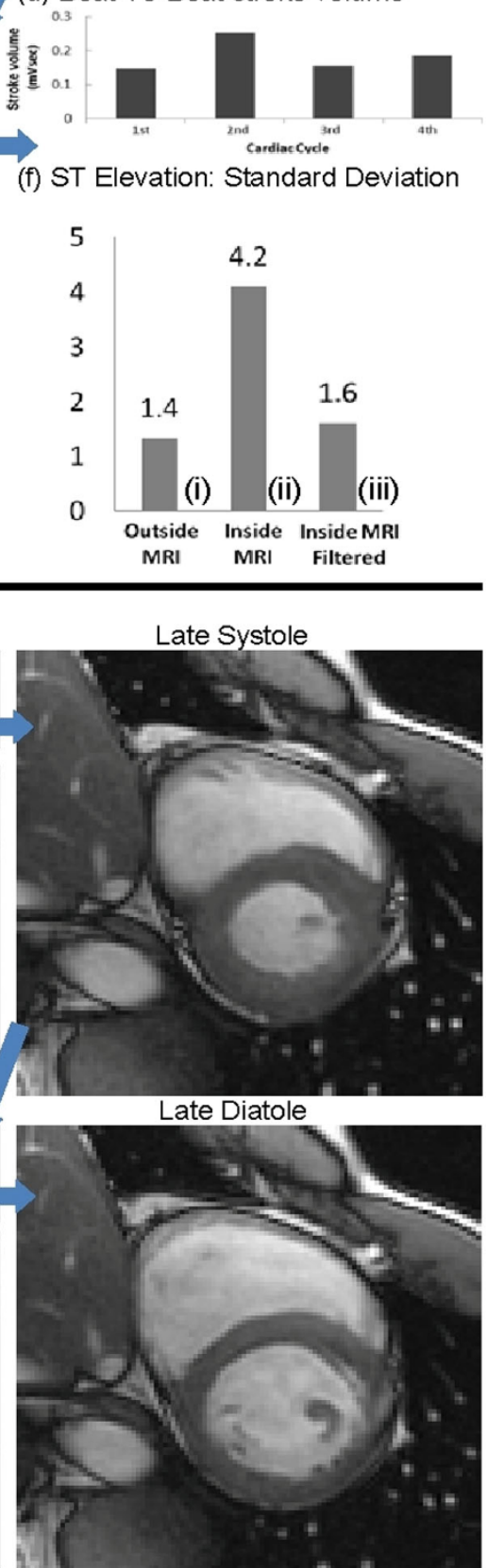

Figure 1 ECG processing of an AF patient in a 1.5T MR. In (b) MR imaging noise is removed. In (c) VMHD is removed \& (d) Stroke volume is provided. (e) The ST elevation mean increased by only $2.5 \%-5 \%(0.062-0.13 \mathrm{mV})$ between ECGs taken (i) outside MRI and (iii) inside MRI with ECG filtered. (f) The corresponding standard deviations are close (1.4-1.6), showing that the filtered ECG taken in (iii) is very close to the true ECG in (i). VMHD in (iii) was effectively removed, extracting real ECG. (g) 12-lead ECG-gated cardiac cine in a subject whose 4-lead ECG gating failed due to a strong MHD peak voltage which eclipsed the QRS complex. 
(a) Swine12-lead ECGs acquired inside MRI at various times post-infarction

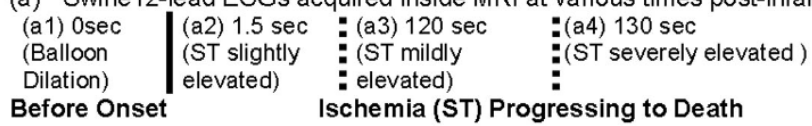

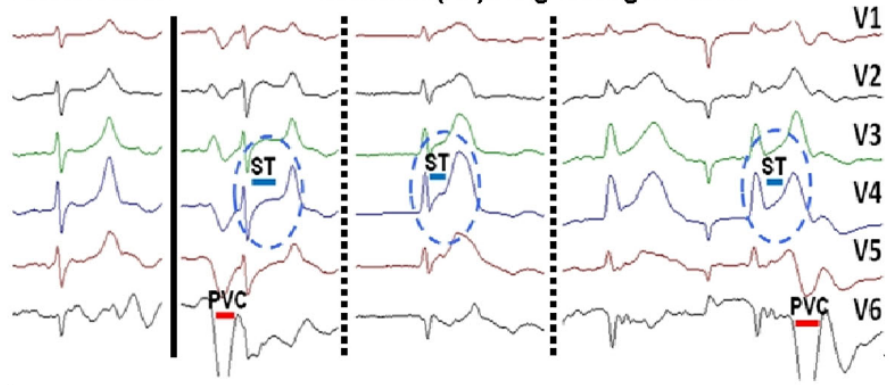

Simultaneous cardiac MRI showing acute ischemia progressing

(b) Inflated Balloon (T1 image)

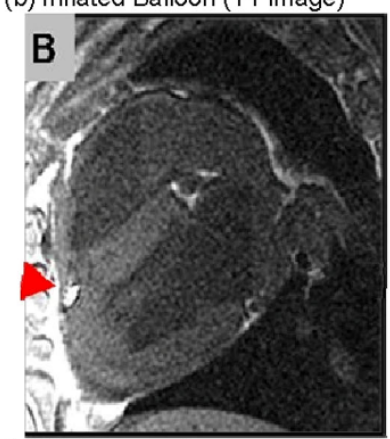

(d) Edema (T2 image)

(c) CINE scan showing irregular flow at right ventricle
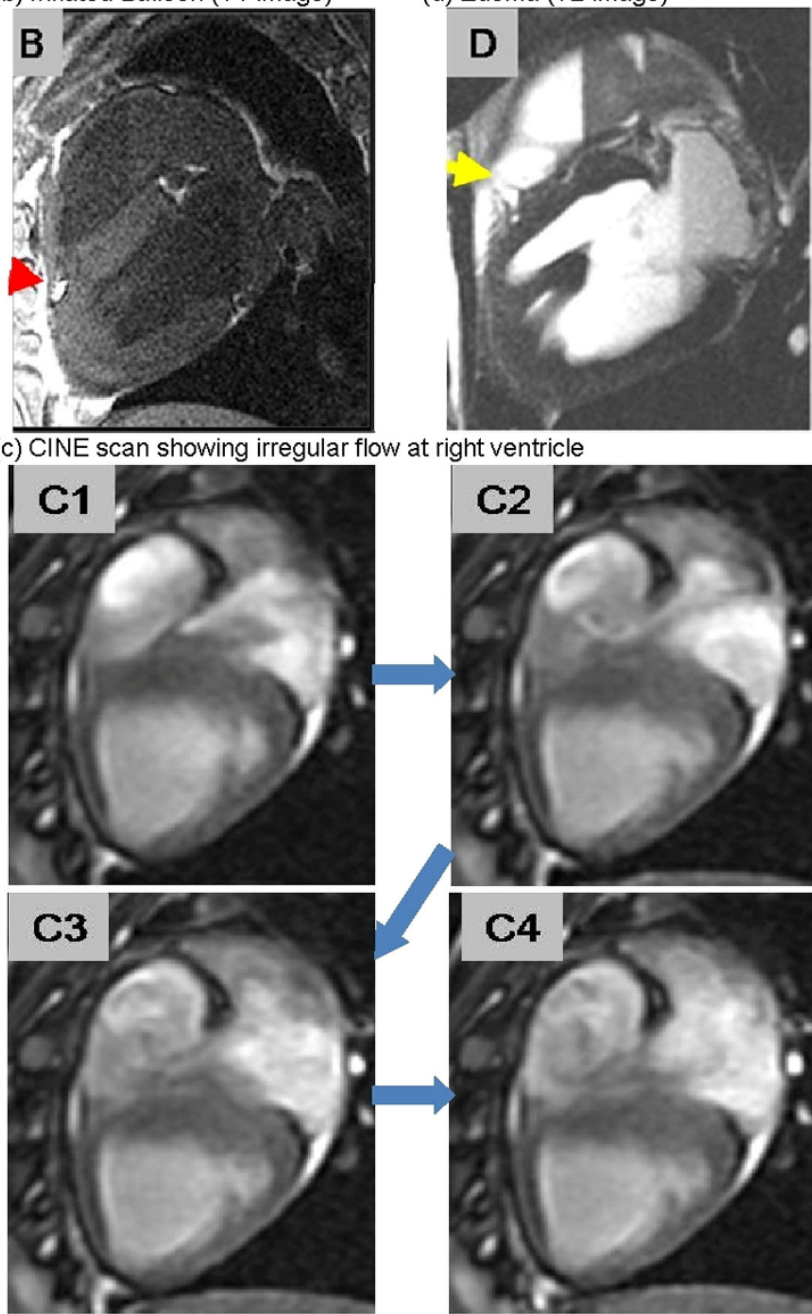

Figure 2 (a) Evolution of ST elevation over time, occasional PVCs and development of bradychardia are clear. (b) Inflated balloon position. (c1-4) Irregular flow patterns during ischemia at $\Delta \mathrm{t}=120-140 \mathrm{sec}$. (d) Epicardial edema proximal to occlusion point. 


\section{Author details}

${ }^{1}$ Engineering, The University of Georgia, Athens, GA, USA. ${ }^{2}$ Radiology,

Cincinnati Children's Hospital Medical Center, Cincinnati, OH, USA.

${ }^{3}$ Radiology, Stanford University, Stanford, CA, USA. ${ }^{4}$ Cardiovascular and

Ablation Technologies, St Jude Medical Inc, St. Paul, MN, USA. ${ }^{5}$ Cardiology,

Brigham and Women's Hospital, Boston, MA, USA. ${ }^{6}$ Radiology, Brigham and

Women's Hospital, Boston, MA, USA.

Published: 30 January 2013

\section{Reference}

1. Tse ZTH:. ISMRM , '09-'11.

doi:10.1186/1532-429X-15-S1-P70

Cite this article as: Tse et al:: Human \& swine studies of concurrent 12-

lead ECG \& MRI. Journal of Cardiovascular Magnetic Resonance 201315

(Suppl 1):P70.

Submit your next manuscript to BioMed Central and take full advantage of:

- Convenient online submission

- Thorough peer review

- No space constraints or color figure charges

- Immediate publication on acceptance

- Inclusion in PubMed, CAS, Scopus and Google Scholar

- Research which is freely available for redistribution

Submit your manuscript at www.biomedcentral.com/submit 